\title{
Women empowering women
}

\author{
Authors: Sarah Faloon, ${ }^{\mathrm{A}}$ May Yan $^{\mathrm{A}}$ and Clare Sarb ${ }^{\mathrm{A}}$
}

\section{Introduction}

Seventy-seven per cent of NHS workers are female, yet only $37 \%$ are in senior positions. ${ }^{1}$ Women have represented the majority of medical school cohorts for over a decade, but this is not translating into increased numbers of women in consultant or leadership roles. Women comprise only $36 \%$ of consultants, $13 \%$ of surgeons and $25 \%$ of medical directors. ${ }^{1-3}$

Gender balance at the top leads to effective financial and quality performance, and there is clear evidence the culture of any organisation is connected to the behaviours of the board and senior leaders. ${ }^{4}$ Women bring unique qualities that are key to the balance of any team.

\section{Materials and methods}

To address this issue a deputy medical director, RCP tutor and core medical trainee at Sandwell and West Birmingham NHS Trust designed a 1-day national free conference aimed at medical female doctors entitled 'Women Empowering Women' to enable, inspire and empower women.

The day involved talks from women in leadership roles, including the president of the Medical Women's Federation, and workshops such as Research and Academia, Management and Leadership and Teaching and Education. Further workshops focused on maternity issues, LTFT working, and self-care. A workshop addressing inappropriate behaviour in the workplace was also very popular. The delegates overwhelmingly reported a day such as this was desperately needed.

The key aspects we aimed to address included:

> role-modeling - showcasing individuals in senior leadership positions and sharing their journey: 'If you can see it, you can be it'

$>$ processes - maternity rights to application of jobs were addressed

> mindset - positivity, the importance of self-care and removing imposter syndrome mentality.

110 women attended, ranging from FY1 to consultant level. A survey was performed to assess how women felt about key issues such as gender balance, applying for senior leadership roles and sexual harassment in the workplace.

Authors: ASandwell and West Birmingham Hospital NHS Trust, Birmingham, UK

\section{Results and discussion}

Highlights of the data showed:

$>25 \%$ had been discouraged to apply for a senior leadership role

$>58 \%$ felt they were not achieving work-life balance. Examples included challenges with childcare, hobbies and self-care

$>70 \%$ felt they were underperforming in their careers due to the many challenges they faced including childcare, illness and exams

$>56 \%$ felt there was a lack of women in senior leadership roles

$>47 \%$ have had sexual comments and inappropriate behaviour from colleagues and seniors in the workplace and $73 \%$ have had this behaviour from patients

$>66 \%$ did not escalate these issues due to fear of potential repercussions or not being taken seriously.

There remains a multitude of issues facing women working in the NHS today. To address this, many initiatives are being generated at out trust including establishing a Women's Network, a leadership mentoring scheme and educational sessions on inappropriate workplace behaviours and how to escalate.

\section{Conclusion}

If we are to tackle issues, such as lack of women in senior leadership positions and sexual harassment in the workplace, then such innovative days must be an embedded feature within each organisation as a positive step forward to rectifying this imbalance.

\section{Conflicts of interest}

None declared.

\section{References}

1 NHS digital. Narrowing of NHS gender divide but men still the majority in senior roles. NHS, 2018. www.digital.nhs.uk/news-andevents/latest-news/narrowing-of-nhs-gender-divide-but-men-stillthe-majority-in-senior-roles [Accessed 09 September 2019].

2 Royal College of Surgeons. Statistics: Women in Surgery. RCS www.rcseng.ac.uk/careers-in-surgery/women-in-surgery/statistics [Accessed 9 September 2019].

3 Rimmer A. Five facts on women in NHS leadership roles. BMJ 2017;357:j1701.

4 Gipson AN, Pfaff DL, Mendelsohn DB, Catenacci LT, Burke WW. Women and leadership: Selection, development, leadership style, and performance. J Appl Behav Sci 2017;53:32-65. 